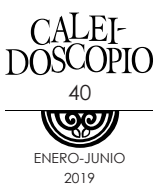

\title{
Normalismo rural y el movimiento del 68. Cañada Honda, Aguascalientes
}

Rural normalism and the movement of 1968. Cañada Honda, Aguascalientes

SERGIO ORTIZ BRIANO'

\section{RESUMEN}

El movimiento universitario de 1968 en México no puede entenderse sin considerar los acontecimientos de esa década alrededor del mundo. Los jóvenes estudiantes se convirtieron en protagonistas de la ruptura del orden establecido. En el presente trabajo nos centramos en el contexto de la normal rural de Cañada Honda, Ags., durante 1968 para identificar algunas preocupaciones expresadas por el normalismo rural a través de la FECSM y de esta escuela en particular, enfocando el nivel de participación de las estudiantes en apoyo al movimiento universitario de 1968. En la realización de este trabajo partimos de que las movilizaciones y el posicionamiento de las normalistas rurales con respecto al conflicto universitario adquieren sentido en tanto que se les aprecia en el contexto del activismo que durante los meses previos venían desarrollando debido a la falta de atención de las demandas propias de esta escuela y las presentadas por su dirigencia nacional.

Palabras clave: normalismo rural, movimiento universitario, FECSM, Cañada Honda.

1 Escuela Normal Rural "Justo Sierra Méndez", México. 
The 1968 university movement in Mexico cannot be understood without considering the events of that decade around the world. The young students became protagonists of the rupture of the established order. In the present work, we focus on the context of the rural normal of Cañada Honda, Ags., during 1968 to identify some concerns expressed by the rural normalism through the FECSM and of this school in particular, focusing on the level of participation of the students in support of the university movement of 1968. In the realization of this work, we start from the fact that the mobilizations and the position of the rural normalistas with respect to the university conflict acquire meaning insofar as they are appreciated in the context of activism that during the previous months they had been developing due to the lack of attention to the demands of this school and those presented by their national leadership.

Keywords: rural normalism, university movement, FECSM, Cañada Honda.

Expresiones contraculturales, libertarias y pacifistas: la reforma universitaria chilena, el mayo francés del 68 y el movimiento universitario del 68 en México, que se da en la antesala de los Juegos Olímpicos, convierten a ese año en una época que hizo temblar al mundo. El conflicto universitario mexicano que tuvo uno de sus momentos más dramáticos el 2 de octubre, tras el asesinato de una cantidad de estudiantes aún desconocida, se enmarca en un contexto de autoritarismo creciente en América Latina que buscaba erradicar toda manifestación con aires de libertad, de tufo comunista o con aspecto de conciencia crítica. Una estrategia de autoritarismo creciente definido por Alain Rouquié como Estados militares articulados por la Doctrina de Seguridad Nacional promovida por Estados Unidos durante la Guerra Fría (Rouquié, 1987, citado en Victoriano, 2010).

Con este antecedente, en el presente trabajo nos centramos en el contexto de la normal rural de Cañada Honda, Ags., para identificar algunas preocupaciones expresadas por el normalismo rural a través de la FECSM y de esta escuela en particular, enfocando el nivel de participación de las estudiantes en apoyo al movimiento universitario de 1968. En la realización de este trabajo partimos de que las movilizaciones y el posicionamiento de las normalistas rurales con respecto al 
conflicto universitario adquieren sentido en tanto que se les aprecia en el contexto del activismo que durante los meses previos venían desarrollando debido a la falta de atención de las demandas propias de esta escuela y las presentadas por su dirigencia nacional.

CONSTRUYENDO EXPEDIENTES. TÁCTICA PARA JUSTIFICAR EL HOSTIGAMIENTO

A pesar del hostigamiento del que venían siendo objeto las escuelas normales rurales durante el primer lustro de los años sesenta, el estudiantado campesino mantuvo con firmeza su dinámica de trabajo e intercambio político. En este contexto, mientras que las escuelas de Saucillo y Salaices, ubicadas en el estado de Chihuahua, iniciaban el año de 1968 con la falta de atención a las demandas presentadas desde el año anterior, como la carencia de maestros, almacenista, tres lavanderas y trabajadores para el campo; ${ }^{2}$ los estudiantes eran acusados de agitadores comunistas (Valdés, 2005, Tomo II). De acuerdo con Valdés, los jóvenes estudiantes cuyas edades oscilaban entre los 12 y los 18 años de edad "eran vigilados constantemente por la policía secreta del estado; por las policías municipales y por policías llegados de la ciudad de México y, además [... ], por espías de la FBI y de la CIA y, peor aún, por soldados del Ejército Nacional" (Valdés, 2005, Tomo II: 307).

En Aguascalientes, a pesar de la aparente calma y de la cálida recepción que las estudiantes de la normal rural de Cañada Honda habían brindado a Gustavo Díaz Ordaz como candidato presidencial el mes de febrero de 1964, ${ }^{3}$ entre los meses de abril y mayo, periodo en el cual se reunieron en esta escuela los representantes de diferentes sociedades de alumnos pertenecientes a la FECSM con la finalidad de buscar medidas que ayudaran a su reunificación, José Santos Valdés ${ }^{4}$ señala:

2 Valdés, José Santos, Visitador Especial de Normales, Informe sobre las visitas a las Escuelas Normales Rurales "Ricardo Flores Magón", sita en Saucillo, Chih., y "Abraham González" de Salaices, Chih., 26, 27, 28 y 29 de octubre de 1967", AGENRISM, caja 33, 1966-1969, José Ma. Morelos, Ags., 10 de enero de 1968.

3 AGENRISM, caja 32, 1965-1967. Discurso pronunciado por la Señorita Ma. Elena Lucio Ortiz del Sexto Año Normal, en la recepción que se ofreció al Lic. Gustavo Díaz Ordaz, en la Escuela Normal Rural "Justo Sierra Méndez", de Cañada Honda, Ags., Cañada Honda, Ags., 10 de febrero de 1964.

4 Inspector de Enseñanza Normal en la Zona Norte de 1960 hasta el 31 de agosto de 1965. Estaban, entre otras, las normales rurales de Cañada Honda, Ags., San Marcos, Zac; Durango, Sonora y Chihuahua.

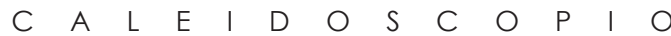


ya no los políticos sino alguna de nuestras múltiples policías, hizo correr la versión de que los líderes de los estudiantes normalistas rurales, reunidos en Cañada Honda, Ags., trataban de organizar y -desde luego llevar a cabo- un movimiento armado en todo el país y que para iniciarlo, para abrir boca -como quien dice-, el $1^{\circ}$ de mayo, aprovechando el desfile obrero en la ciudad de Aguascalientes, provocarían un motín sangriento (Valdés, 2005, Tomo II: 311 ).

Coincidentemente, en un hecho que concuerda con la hipótesis del hostigamiento, el director de la normal rural de Cañada Honda le informaba al procurador general de Justicia del estado de Aguascalientes que, desde las últimas semanas del mes de febrero y los primeros días del mes de marzo de 1964, empezaron a vivirse "algunos hechos fuera de lo normal [...] dentro del perímetro de esta institución". ${ }^{5}$ Entre otros, le aclaraba que "con mucha frecuencia transita por los linderos de la Escuela un vehículo no identificado sin placas, color negro, a altas horas de la noche [...] Se ha visto rondar en las proximidades de la Escuela y en horas avanzadas de la noche a individuos desconocidos que posiblemente transitan en el vehículo de referencia". ${ }^{\circ}$

Aunque no sucedió lo que se había previsto para el desfile del $1^{\circ}$ de Mayo en Aguascalientes, un día después de este evento las autoridades justificaban sus predicciones señalando que habían tenido razón, ya que un "grupo de normalistas rurales de San Marcos -con su banda de guerra- había desfilado a la cabeza de los obreros ferrocarrileros vallejistas [...]" (Valdés, 2005, Tomo II: 312). Lo que desde este punto de vista significaba una alteración del orden social. Y la zozobra continuó; más adelante sucedió con los normalistas rurales de Roque, en Guanajuato, a quienes se les acusó de preparar un levantamiento armado para el mes de julio, particularmente el domingo en que se llevarían a cabo las elecciones presidenciales. Al igual que en otros casos, la situación fue desmentida por los propios políticos y pobladores de esa región.

5 Oficio dirigido al Lic. Manuel Ávila Salado, Procurador General de Justicia del Estado en el que "Se ponen en conocimiento anomalías que acontecen por las noches en la Escuela". Silverio Díaz Cárdenas, Director de la Escuela Normal Rural de Cañada Honda. AGENRJSM, caja 30, 1960-1964, Sección Correspondencia, Oficio Número 328, 12 de marzo de 1964.

6

Idem. 
Las afirmaciones alrededor de los normalistas rurales, tal como sucedió con aquellos grupos o personas con alguna inquietud opuesta al gobierno, parecen contener la finalidad de construir expedientes que justificaran su persecución. En este sentido, puede considerarse que estos expedientes responden a intereses represivos, si se piensa que, además del control de la prensa por parte del gobierno, también se contaba durante esos años, 1958-1969, con el involucramiento de la CIA en México. Baste mencionar, como un ejemplo, el caso descrito por Morley Jefferson citado por Aguayo (2018) acerca de Víctor Rico Galán, que por tratarse de "un periodista crítico muy conocido por aquellos años. Según Morley, la CIA ayudó a armar el caso y en septiembre de 1966 fueron detenidos |Rico Galán| y 28 asociados. El periodista se pasaría siete años en Lecumberri" ${ }^{7}$

\section{CAÑADA HONDA. ENTRE EL EJERCICIO CORPORATIVO Y MONEDA DE INTERCAMBIO POLÍTICO}

Durante esos años, las sociedades de alumnos de las normales rurales se mantuvieron en actividad constante alentando el fortalecimiento de su unidad y su fraternidad y, por supuesto, en su postura de manifestación y exigencia de todas aquellas demandas que desde su punto de vista contribuían a mantener la vigencia de sus instituciones. Aspectos sustanciales en la reivindicación de su organización estudiantil en el contexto político del siglo Xx. Algunos espacios de intercambio generados entre los estudiantes campesinos fueron los festejos de aniversario de algunas de estas escuelas; las llamadas Jornadas Culturales y Deportivas que se realizaban anualmente de manera itinerante en diferentes sedes; así como la presentación de pliegos de peticiones y su participación en movimientos estudiantiles desarrollados para exigir la satisfacción de sus demandas o en solidaridad con otras organizaciones de estudiantes.

En tanto que la FECSM se encargó de organizar y encabezar algunas de las manifestaciones estudiantiles durante esos años a nivel nacional, las sociedades de alumnos hicieron lo propio al exigir a las autoridades de las entidades la atención de sus demandas. Sin em-

7 Jefferson, Morley, Our Man in Mexico. Winston Scott and the Hidden Story of the CIA, Citado en Aguayo Quezada, Sergio (2018), EL 68. Los estudiantes, el presidente y la CIA. Ediciones Proceso, México, p. 9.

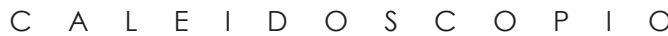


bargo, podemos apreciar que en el caso de la Normal Rural de Cañada Honda, el activismo de las estudiantes estuvo entre la disciplina impuesta por su organización estudiantil y sus intereses locales. De tal manera que mientras por un lado se involucran en actividades relacionadas con la dinámica de participación política impuesta por la FECSM en defensa de estas escuelas, por otro lado muestran su regocijo por la presencia de políticos y funcionarios en las instalaciones de su escuela.

Los preparativos para la visita que realizaría Díaz Ordaz durante los primeros días de febrero de 1964 al estado de Aguascalientes permiten apreciar el sentido corporativo de las instituciones y su alianza directa con el partido oficial, así como algunos elementos contradictorios con respecto de la postura revolucionaria del normalismo rural. En medio de un ambiente laboral que vivía las consecuencias del conflicto que desmanteló al grupo de seguidores de Demetrio Vallejo, los líderes ferrocarrileros expresaron su preocupación por representar los intereses del gremio y por generar más plazas laborales en estos talleres al proponer el establecimiento de una fábrica de vagones para pasajeros; pero reconociendo que "todos los sectores deben unificarse para hacer esa petición a Díaz Ordaz" ${ }^{8}$

Los sectores campesino y magisterial también hicieron pública su entrega y regocijo ante la visita del candidato. El magisterio de Aguascalientes, asimismo, decía unirse "al regocijo del Pueblo de Aguascalientes para dar cordial bienvenida al Lic. Gustavo Díaz Ordaz". En este ambiente, todas las presentaciones del candidato en los diversos puntos del estado se vieron favorecidas con la presencia de grandes "contingentes de escolares y ciudadanos que asistieron espontáneamente" ${ }^{10}$ Como parte de estas presentaciones el domingo 9 de febrero Gustavo Díaz Ordaz estuvo en la Normal Rural de Cañada Honda. ${ }^{11}$

8 "Debe insistirse en la fábrica de coches de ferrocarril", en El Sol del Centro, Domingo 9 de febrero de 1964, AHEA.

9 Idem.

10 "A las 11:40 llegó a ésta el Lic. Díaz Ordaz", en El Sol del Centro, lunes 10 de febrero de 1964, AHEA.

11 De acuerdo con las "Actividades que Desplegará Hoy el Lic. Díaz Ordaz", se aprecia que a las 15:30 horas llegaría a la Escuela Normal de Cañada Honda. Antes de regresar a la ciudad capital en donde a las 20:00 horas se ofrecería una Fiesta Popular en la Plaza en su honor, además de participar en una "cena que ofrecerán los ganaderos de Aguascalientes". En El Sol del Centro, lunes 10 de febrero de 1964, AHEA. 
Pero, ¿cómo es que si los normalistas rurales se reconocían perseguidos por el gobierno, las estudiantes de esta escuela hayan recibido con muestras de cordialidad tanto al gobernador del estado como al candidato oficial a la presidencia de la República? Aunque resulta difícil conocer los elementos tomados en cuenta por las estudiantes para aceptar la cercanía con las autoridades locales y federales a pesar de la situación en que estaba envuelto el normalismo rural, algunas hipótesis apuntan a explicar ese hecho tanto a partir de la veneración que por aquella época se tenía al cargo de presidente ${ }^{12}$ como en la relación construida con el gobierno del estado de Aguascalientes por iniciativa de su titular, el profesor Enrique Olivares Santana, advertida como una estrategia de las estudiantes para desviar la mirada de la autoridad al concretarse el cierre de las estas instituciones con la reforma de educación normal de 1969. Recordemos que, a pesar de que durante esos años estuvieron atentas a las convocatorias de su organización estudiantil, con el inicio de la década de los años sesenta entraron en una dinámica de relación estrecha con Enrique Olivares Santana, quien acudía a visitarlas con cualquier motivo y "se encargó de resolver cualquier petición que le hiciéramos, cualquier necesidad de la escuela" (Barba Campos, 21 de mayo del 2018, entrevista).

Se puede decir que la presencia de Gustavo Díaz Ordaz en la normal rural de Cañada Honda se debió a la cercanía que no gratuitamente fue construyendo Enrique Olivares Santana como gobernador constitucional de la entidad con las estudiantes. De acuerdo con Vital Díaz, desde 1963 "le había organizado al entonces candidato presidencial Díaz Ordaz una gira por Cañada Honda, antigua hacienda donde ahora estaba una Normal y donde el futuro Presidente convivió con la Federación de Estudiantes Campesinos Socialistas de México. La comunidad era pequeña y funcional; el abanderado priísta estuvo contento" (Vital Díaz, 2006: 223).

12 De acuerdo con Aguayo, las escasas encuestas de aquella época señalan que la mayor parte de la población veneraba al presidente, respetaba su autoridad y tenía una paupérrima comprensión del mundo (2018: 22), además, según el Instituto Mexicano de Opinión Pública, "antes de Tlatelolco, 60 por ciento de los capitalinos pensaba que el presidente tenía buena comprensión (empatía) con una población que lo consideraba un padre enérgico pero justo". 1968. Encuesta realizada por el Instituto Mexicano de Opinión Pública, sin fecha. AGN. Fondo Gobernación, Sección DGIPS, caja 1463, citado en Aguayo, 2018: 97. 
Años después, cuando el gobierno de Olivares Santana se había consolidado y cuando había dado muestras de sus habilidades políticas, no sólo tuvo el reconocimiento de Díaz Ordaz, quien recomendó "a los gobernadores que se dieran una vuelta por Aguascalientes, en donde las cosas estaban marchando como a él le gustaban", sino que además de realizar una segunda visita a esta escuela el 7 de febrero de $1967,{ }^{13}$ tiempo después recibió la invitación para "ser el secretario general del partido a muy poco menos de un año de que concluyera" (Vital Díaz, 2006: 225) su mandato como gobernador.

Así, ante la visita del Lic. Gustavo Díaz Ordaz en febrero de 1964, las estudiantes expresaron su alegría por lo que definieron como una verdadera muestra de sencillez del candidato presidencial al visitarlas para conocer sus necesidades y sus problemas, "en la fuente misma de su realización en la vida cotidiana". ${ }^{14}$ En esa ocasión, aparte de reiterarle la esperanza que veían en su persona, las estudiantes le señalaron su compromiso para devolverle al "pueblo que nos dio la oportunidad de estudiar, nuestros servicios profesionales y nuestra lealtad a sus nobles causas". ${ }^{15}$ Además, en un mensaje que muestra la ideología ya característica de estas instituciones, le garantizaban que seguirían en su compromiso heredado en todas "nuestras Escuelas Normales Rurales, 29 en su totalidad, [las cuales] han tenido una trayectoria grandiosa, han rendido frutos a través de los miles y miles de maestros que por todos los rumbos de la Patria, llevan la luz del alfabeto, y el mensaje ideológico de la Revolución". ${ }^{16}$

13 AGENRISM, caja 32, 1965-1967, Discurso pronunciado por la Señorita Graciela Reyes Martínez, con motivo de la recepción que se le tributó al C. Lic. Gustavo Díaz Ordaz, en la Escuela Normal Rural "Justo Sierra Méndez", de Cañada Honda, Ags., 7 de febrero de 1967.

14 Lucio Ortiz, Ma. Elena, AGENRISM, caja 31, 1963-1966, Discurso pronunciado en la recepción que se ofreció al Lic. Gustavo Díaz Ordaz, en la Escuela Normal Rural "Justo Sierra Méndez" de Cañada Honda, Aguascalientes 10 de febrero de 1964.

15 Idem

16 Idem. Al referirnos a la ideología en el normalismo rural estamos aludiendo a la postura de lucha y exigencia de los estudiantes ante las autoridades por la mejora de las condiciones de sus internados y la mejora en la calidad de su formación como futuros maestros y maestras rurales. En este sentido, reconocen como hermanos en ideales a los "Estudiantes Campesinos de la República y a los abnegados Maestros Rurales del País". Vanguardia Campesina, Órgano de la Escuela Regional Campesina de Roque, 1935, Guanajuato. AHSEP, Fondo DEANR, Guanajuato, Caja 4, Expediente X/121.4 (x-5) (724.4)/-2. 
En su segunda visita, en donde además lo volvía a acompañar el profesor Enrique Olivares Santana, la alumna encargada del mensaje de bienvenida replica el patrón de apologías hacia este personaje empleadas tres años antes. De entrada, recuerda que en febrero de 1964 esta escuela "tuvo el alto honor de abrir sus puertas y los corazones de esta comunidad escolar para tributarle el más caluroso homenaje, en ocasión de su visita como candidato del pueblo de México, a la Primera Magistratura". ${ }^{17}$ Por otro lado, al recordarle que reconocen su condición de "estudiantes revolucionarios" también le expresaban: "Hoy vestimos esta Institución Educativa con los colores de nuestro sacro lábaro, para rendirle nuestra más fervorosa bienvenida en la alta investidura que la Patria ha puesto en sus manos". ${ }^{18}$

NORMALES RURALES. FABRICACIÓN DE EXPEDIENTES Y APOYO AL MOVIMIENTO UNIVERSITARIO

A pesar de este contexto, pero siguiendo la táctica de fabricación de expedientes, las manifestaciones de inquietud expresadas por las normalistas rurales por la falta de atención de sus demandas en mejora de su preparación como futuras maestras rurales en la coyuntura del movimiento universitario fueron aprovechadas por la autoridad para mostrarlas como "responsables de la pérdida de tiempo tan necesario en la buena preparación de los futuros maestros"19 y con esto justificar la reorganización de las escuelas de este tipo como parte de la implantación de la Reforma de Educación Normal de 1969.

La situación de las normales rurales coincidía con la crisis que venían enfrentando los universitarios. En cuanto a la crisis universitaria

17 AGENRJSM, caja 32, 1965-1967, Discurso pronunciado por la Señorita Graciela Reyes Martínez, alumna del primer grado profesional, con motivo de la recepción que se le tributó al C. Lic. Gustavo Díaz Ordaz, en la Escuela Normal Rural "Justo Sierra Méndez de Cañada Honda, Ags., 7 de febrero de 1967.

18 AGENRISM, caja 32, 1965-1967, Discurso pronunciado por la Señorita Graciela Reyes Martínez, alumna del primer grado profesional, con motivo de la recepción que se le tributó al C. Lic. Gustavo Díaz Ordaz, en la Escuela Normal Rural "Iusto Sierra Méndez de Cañada Honda, Ags., 7 de febrero de 1967.

19 Caja 34, Dirección General de Enseñanza Normal, Oficio 12656, A los padres de familia y estudiantes de la Sociedad de Alumnas de la Escuela Normal Rural de Cañada Honda, Ags., 18 de noviembre de 1968.

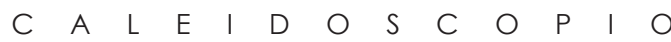


cabe decir que luego de la persecución de los granaderos a estudiantes de las vocacionales 2 y 5 del Instituto Politécnico Nacional ocurrida el 22 de julio de 1968, las marchas de protesta ocurridas el día 26 y posteriores dieron lugar a la alianza Politécnico-UNAm para convertirlo en un Movimiento Estudiantil de grandes dimensiones, cuya bandera principal era la lucha por la autonomía universitaria y un pliego petitorio que incluía la libertad de todos los presos políticos, destitución de jefes policiacos y desaparición del cuerpo de granaderos. Los normalistas rurales, por su parte, continuaban en su lucha por la satisfacción de sus demandas históricamente desatendidas, como la alimentación, la creación y equipamiento de espacios para su preparación, o la falta de personal adecuado para la formación de maestros.

Es en este contexto en donde además de las luchas constantes que venían enfrentando los normalistas rurales en la demanda de atención a sus propias preocupaciones a lo largo de 1968, también hicieron visibles sus manifestaciones de apoyo al movimiento universitario. Fue desde un mes antes y aun después de los sucesos de la noche más triste de Tlatelolco cuando los estudiantes campesinos realizaron diferentes actividades para condenar públicamente las agresiones del gobierno y mostrar su solidaridad con el movimiento universitario.

A partir del último día del mes de febrero de 1968, la Sociedad de Alumnas "Amalia Solórzano de Cárdenas", de Cañada Honda, inició una huelga con la finalidad de exigir la atención a las demandas que consideraban como necesarias para continuar con una formación que les permitiera estar a la altura de la superación que exigía el pueblo mismo. De acuerdo con los comunicados dirigidos a la dirección de la escuela, para las estudiantes de Cañada Honda resultaba por demás claro el interés de las autoridades educativas porque "el estudiantado rural tenga una incompleta preparación, negando los medios necesarios que se requieren para la superación de éste y del pueblo mismo". ${ }^{20}$ Una superación que "no terminará hasta que sus problemas sean resueltos favorablemente". ${ }^{21}$ Sin embargo, para llegar a esa situación fue necesario pasar por un proceso de paros escalonados de actividades entre los que se encuentran el anunciado de 24 horas para el día 17 a

20 Comunicando realización de paro de actividades con duración de 48 horas, a la Dirección de la Escuela. Sociedad de Alumnas "Amalia Solórzano de Cárdenas", Cañada Honda, Aguascalientes, 18 de febrero de 1968

21 Idem. 
partir de las 6:00 horas; uno más de 48 horas a partir del día 21 a las 6:00 horas, mismo que no concluyó y que, en todo caso, al día siguiente fue declarado otro paro, pero ahora con duración de setenta y dos horas. $^{22}$

La estrategia utilizada por la autoridad educativa en esa ocasión fue la más socorrida durante ese sexenio: exhibir a las estudiantes ante la sociedad como intransigentes. Es decir, mientras que "los órganos interesados en desvirtuar el movimiento" 23 venían informando que habían sido resueltas "más del $80 \%$ de las citadas peticiones", ${ }^{24}$ los padres de familia conscientes de la situación sentenciaban que "sólo un tonto podría creer que un triunfo de tal naturaleza sería rechazado por los estudiantes, a menos de que fueran unos intransigentes como se pretende exhibirlos". ${ }^{25}$ Ahora bien, con el involucramiento de los padres de familia que empezaron a organizarse "para ver la manera de ayudar a nuestras hijas en la lucha que han emprendido por el mejoramiento de sus escuelas y de la educación que reciben", ${ }^{26}$ es muy probable que la autoridad educativa haya valorado la dimensión que comenzaba a adquirir el movimiento y el conflicto finalmente se resolvió. ${ }^{27}$

No sería sino hasta meses después cuando, sin abandonar sus peticiones particulares, la Sociedad de Alumnas "Amalia Solórzano de Cárdenas", al considerar que "el movimiento estudiantil tiene la finali-

22 Se transcribe oficio de la Sociedad de Alumnas de esta Escuela, dirigido al Director General de Enseñanza Normal. Cañada Honda, Aguascalientes, 16 de febrero de 1968; Comunicando realización de paro de actividades con duración de 48 horas, a la Dirección de la Escuela. Sociedad de Alumnas "Amalia Solórzano de Cárdenas", Cañada Honda, Aguascalientes, 18 de febrero de 1968; y Telegrama Oficial, dirigido a Ramón G. Bonfil, Director General de Enseñanza Normal. Vía Cañada Honda, Aguascalientes, 22 de febrero de 1968.

23 Circular Número 2. Citatorio a los compañeros Padres de Familia. Miembros de esta Sociedad. Comité Ejecutivo de la Sociedad de Padres de Familia, Cañada Honda, Aguascalientes, 6 de marzo de 1968.

24 Idem

25 Idem

26 Idem.

27 Telegrama Oficial. Infórmole actividades escuela a mi cargo coma desarróllanse normalmente. Dirigido a la Dirección General de Enseñanza Normal. Cañada Honda, Aguascalientes, 11 de marzo de 1968.

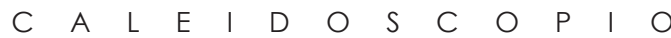


dad de una verdadera democracia para nuestro pueblo", ${ }^{28}$ anunciaba la realización de un paro de actividades de 12 horas para el siguiente día para protestar contra la intervención del ejército en los centros educativos y para reprobar la omisión de las autoridades ante esos hechos, por lo que exigía, además, la solución a los problemas presentados por la juventud universitaria. En este sentido, Daniel Carlos García ha señalado que los normalistas rurales de San Marcos, Zac., y de Cañada Honda, Ags., ya desde finales del mes de agosto y durante la primera mitad de septiembre, además de mostrar actitudes de solidaridad frente al conflicto estudiantil desencadenado en la ciudad de México desde el 22 y 23 de julio, habían realizado una importante labor de difusión entre la población aguascalentense después de la acción policíaca contra las manifestaciones del 26 de julio (García, 2006).

\section{MOVILIZACIÓN ESTUDIANTIL. REIVINDICAR EL NORMALISMO RURAL}

A pesar de las características de la comunicación de la época, las normalistas rurales pronto fueron enteradas de los acontecimientos del miércoles 2 de octubre en la ciudad de México. En este sentido, mientras que Mercedes Palomino recuerda que participaron "en guardias $[\ldots]$ estar en los patios, platicando, mientras hacíamos guardia [...]. Recuerdo una noche de esas estar haciendo guardia arriba, en el torreón, al pendiente de dar aviso en caso de ver algo extraño. Que alguien se quisiera meter, pues avisar. Pero no pasó nada". ${ }^{29}$ Élida Hernández González, quien formaba parte de la dirigencia estudiantil de esta normal rural, recuerda que todavía días después una compañera fue a la Ciudad de México y trajo algunas revistas que narraban los sucesos y ella misma complementaba las pláticas de un estudiante universitario que estuvo en Cañada Honda. "Y bueno, en el movi-

28 AgEnRJSm, caja 34, 1968-1970, Sociedad de Alumnas "Amalia Solórzano de Cárdenas", Paro de Actividades de 12 horas, contra la intervención del ejército en los Centros Educativos. Con firmas de Evangelina Félix Villa como Secretaria General y Élida Hernández González, Secretaria de Actas y Acuerdos. También, Escuela Normal Rural "Justo Sierra Méndez", Se transcribe oficio de la Sociedad de Alumnas. Cañada Honda Aguascalientes, 19 y 20 de septiembre de 1968, respectivamente.

29 Palomino González, Mercedes (1964-1970). Entrevista realizada el 19 de mayo del 2018 en Villa Juárez, Ags., por Sergio Ortiz Briano. 
miento del 68 sí se participó pero no así tan directamente [...]. Porque en aquel tiempo la FECS (sic) tenía una sede en México [...]. Entonces aunque nosotras no íbamos, eran nuestros representantes los que participaban. Pero nosotras lo hicimos desde acá". ${ }^{30}$

Durante los días posteriores a los trágicos acontecimientos de Tlatelolco, los normalistas rurales participaron, hasta donde les fue posible, en diversas manifestaciones. Una de éstas sucedió el 3 de octubre, cuando de acuerdo con un diario local se reunió un grupo de estudiantes que no llegaban a doscientos. Entre ellos venía un grupo de estudiantes procedentes de La Huerta, Mich.; algunos de San Marcos, Zac.; las alumnas de Cañada Honda, Ags.; "una doctora y un arquitecto procedentes de la capital de la República, según se supo, fueron quienes organizaron la manifestación". ${ }^{31}$ El mismo diario aclara que la manifestación se realizó con un recorrido por algunas calles del centro de la ciudad en donde además del Himno Nacional lanzaron consignas como: ¡Prensa Vendida, Prensa Vendida!; ¡Libros sí, bayonetas no! Culminando con un mitin en la exedra, en donde, de acuerdo con el propio diario, participaron algunos infiltrados en el motín cuyos discursos eran propios de filomarxistas. Se trata de "seis oradores: dos estudiantes de la Normal Rural de San Marcos, Zac; una joven estudiante de la Normal de Cañada Honda, un universitario, un estudiante michoacano y un alumno del Instituto de Ciencias". ${ }^{32}$

Como se menciona en páginas anteriores, la participación de las normalistas rurales se dio en el contexto de sus propias preocupaciones. Así se aprecia en un oficio de la Sociedad de Alumnas anunciando un paro de actividades de 36 horas a partir de las 6 horas del 8 de octubre y hasta las 18 del siguiente día, en vista de que las peticiones del estudiantado universitario "para bien de nuestro pueblo" no habían sido atendidas; pero también para exigir "el cumplimiento de los acuerdos tomados en el movimiento de Huelga próximo pasado del Sistema Normal Rural". ${ }^{33}$ Fue también en los mítines realizados en

30 Hernández González, Élida. Entrevista. 8 de septiembre del 2007, Sergio Ortiz.

31 AHEA, "Manifestación Estudiantil en Apoyo del Movimiento del D.F.". El Sol del Centro. Año XXIV. Número 8430. Aguascalientes, Ags., viernes 4 de octubre de 1968.

32 Idem.

33 AGENRISM, caja 34, 1968-1970. Sociedad Estudiantil "Amalia Solórzano de Cárdenas". Escuela Normal Rural "Justo Sierra Méndez". Oficio Número 13, El que a continuación se indica, Cañada Honda, Ags., a 8 de octubre de 1968. 
donde, además de exigir el esclarecimiento de los acontecimientos y el respeto a la integridad del estudiantado en general, las alumnas hicieron patente su exigencia por la atención de sus propias demandas.

A pesar de esto, la participación de las normalistas rurales adquiere particular importancia si se toma en cuenta que en Aguascalientes no existieron otros grupos sociales con la iniciativa para expresar algún tipo de apoyo al movimiento universitario, lo que finalmente contribuía en la reivindicación del estudiantado campesino como un importante grupo ideológicamente radical y crítico del gobierno.

\section{INOUIETUDES ESTUDIANTILES Y MEDIDAS DE CONTROL}

Un tema por demás interesante es que, luego de que durante los movimientos organizados por la FECSM entre 1967 y 1968, en muchos de los casos fueron suspendidos todos los servicios al interior de estas escuelas, lo que obligó a sus estudiantes a salir a las calles a botear para hacerse de recursos; las egresadas entrevistadas coinciden en señalar que en Cañada Honda esto no sucedió gracias al apoyo de Enrique Olivares Santana, "que no permitió que nos cortaran la alimentación. Él absorbió esos gastos". ${ }^{34}$ Sin embargo, esto que se advierte como una actitud de bondad y empatía del mandatario estatal para con las estudiantes, en la práctica parece tratarse de una estrategia para deslegitimar las inquietudes normalistas frente a la opinión pública. De tal manera que, a pesar de que la participación de las normalistas rurales estaba, según la percepción de las estudiantes de la época, autorizada por el gobierno del estado, no por eso estuvo exenta del descrédito y la amenaza que, a través de los diarios locales y de las disposiciones oficiales, pretendieron controlar al estudiantado.

Si en la Ciudad de México se reconoce la presencia de agentes infiltrados o de policías encubiertos para generar actos violentos que permitieran dar a la opinión pública la idea de que los acontecimientos estaban siendo orientados por agitadores comunistas (Guevara, 2004), en Aguascalientes ocurre algo similar al acusar a los estudiantes de utilizar un discurso propio de filomarxistas; pero también, pre-

34 Barba Campos, Arminda Generación (1961-1968). Entrevista realizada por Sergio Ortiz Briano en la Normal Rural de Cañada Honda, Aguascalientes el 21 de mayo del 2018 
tendiendo generar una opinión negativa en la sociedad, al señalarlos como jóvenes sucios, mugrosos que visten con harapos y que al protestar imponen su vandálica, autoritaria y dictatorial autoridad de impedir el paso a quienes transitan libremente y quitan el legítimo derecho a estudiar a quienes desean asistir a clase y salvar su año. ${ }^{35}$

Por otro lado, El Sol del Centro, congruente con su imaginario conservador y desde su trinchera anticomunista, los días posteriores a los acontecimientos del 2 de octubre publicó editoriales cuya finalidad era deslegitimar al movimiento universitario y a aquellos que se movilizaran en su apoyo. Para esto, haciendo uso de las declaraciones de un líder estudiantil integrante del Consejo Nacional de Huelga de dudosa reputación, que más adelante sería identificado como infiltrado en el movimiento, habla de la injerencia de intereses comunistas en México que buscaban crear un "estado de obreros y campesinos de tipo comunista" ${ }^{\prime 6}$ y el arsenal decomisado en el centro habitacional de Tlatelolco a los "jóvenes idealistas del movimiento estudiantil por la fuerza pública tras de los sangrientos sucesos del 2 de octubre". ${ }^{37}$

Por otro lado, aunque las movilizaciones organizadas por la FECSM se dieron a conocer a la luz pública como carentes de relación con respecto de la iniciativa del gobierno de concretar la reforma educativa de las escuelas normales -mostrándola en todo caso como una expresión más de la rebeldía juvenil de la época o como manifestaciones alentadas por el movimiento universitario, a través de la correspondencia mantenida durante estos meses entre las autoridades y la representación estudiantil de la FECSM, y de ésta a su vez con todas las normales rurales del país-, se puede apreciar que con todo y la falta de difusión en torno del cambio en la política educativa, los sucesos desencadenados fueron las primeras muestras de oposición y rechazo ante lo que, de acuerdo con los alumnos de estas escuelas, sería inevitable: el cierre de las normales rurales.

35 AHEA, "Protesta Contra la Protesta". El Sol del Centro. Año XXIV. Número 8430. Aguascalientes, Ags., viernes 4 de octubre de 1968.

36 AHEA, "Editorial". El Sol del Centro. Año XXIV. Número 8433. Aguascalientes, Ags., lunes 7 de octubre de 1968.

37 "Texto de la Declaración de Campos Lemus", en Knochenhauer, María de los Ángeles (comp.), El movimiento estudiantil en México, México, Documentos, citado en Aguayo (2018: 85). También "Editorial". El Sol del Centro. Año XXIV. Número 8434. Aguascalientes, Ags., martes 8 de octubre de 1968. AHEA.

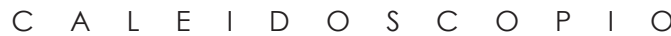


Al igual que en febrero de ese año, durante los últimos días del mes de septiembre y hasta los últimos de noviembre, los normalistas rurales coordinados por la dirigencia nacional de la FECSM se mantuvieron en una constante lucha, situación que permite apreciar la capacidad que las normales rurales, tanto como las universidades mostraron para convertirse en antenas que expresaban la ola de descontentos de la época. ${ }^{38}$ Situación que da cuenta de cómo las movilizaciones realizadas por los normalistas rurales durante ese año respondieron tanto a la inercia de las inconformidades surgidas en el seno de estas escuelas como a las problemáticas desencadenadas alrededor del movimiento universitario.

Una de las medidas de control implementadas por la autoridad que ayuda a inferir su preocupación por el crecimiento del conflicto estudiantil del normalismo rural durante ese año y que lo relacionaba con los universitarios fue el mensaje enviado a los directores a través del cual les pedía recordarles a las estudiantes "que su calidad de alumnas de ese plantel implica la responsabilidades de las autoridades escolares [y que] su responsabilidad como director cesa en absoluto cuando las alumnas abandonan la institución solas o en grupos sin autorización o cuando se dediquen a actividades ajenas a lo específico de estudiantes". ${ }^{39}$

Como se observa a través de este telegrama, el interés de la autoridad por "evitar al ejército la pena -que mucho lamenta- de que se produzcan muertos y heridos de una y otra parte", ${ }^{40}$ generó que los días y semanas posteriores a la noche de Tlatelolco las autoridades también mantuvieron una estrecha vigilancia de los normalistas rurales y lo hicieron desde diversos frentes. Por un lado, dando a conocer la res-

38 A pesar de que desde la percepción del gobierno (basado en los servicios de inteligencia mexicanos y extranjeros) la sociedad estaba bien controlada, "entre noviembre de 1963 y junio de 1968 hubo al menos 53 revueltas estudiantiles en México". Memorándum de AmEmbassy Mexico a Departamento de Estado, "Review of Student Disturbances in Mexico in Recent Years", 23 de agosto de 1968, POL 13-2 MEX, Archivos Nacionales, Washington, citado en Aguayo, 2018: 22.

39 AGENRISM, caja 34, 1968-1970, Escuela Normal Rural de Cañada Honda, Sección Correspondencia, Se transcribe telegrama número E-10484 de la Dirección General de Enseñanza Normal. Al Comité Ejecutivo de la Sociedad de Alumnas de esa Institución. Cañada Honda, Aguascalientes, a 28 de octubre de 1968.

40 AHEA, "Exhortación del Secretario de la Defensa”. El Sol del Centro. Año XXIV. Número 8429. Aguascalientes, Ags., jueves 3 de octubre de 1968. 
puesta a promesas antes hechas o brindando los apoyos más demandados por los estudiantes campesinos, pero también, pretendiendo dividir al estudiantado al señalar la cercanía de algunos estudiantes con autoridades. ${ }^{41}$ Por ejemplo, en correspondencia fechada el 4 de octubre de ese año el propio director general de Educación Normal reconoció que el secretario de Educación habría conseguido inicialmente "la cantidad de once millones de pesos para adaptación y acondicionamiento de las Escuelas Normales Rurales [cantidad que posteriormente] fue aumentada hasta treinta y dos millones de pesos". ${ }^{42}$

\section{CONCLUSIONES}

Las convulsiones de los años sesenta, donde los estudiantes universitarios tuvieron un protagonismo importante, también permiten reconocer que, a pesar de que los normalistas rurales se involucraron de diferentes maneras en este conflicto, lo cierto es que más que formar parte del movimiento universitario del 68, ellos vivieron su propio sesenta y ocho. En este caso, los expedientes que se mencionan en torno a la situación que venían enfrentando las escuelas normales rurales de Salaices y Saucillo, Chihuahua; Roque, Guanajuato y Cañada Honda, Aguascalientes, parecen encerrar un anuncio del tipo de atención que les daría el gobierno a las problemáticas de estas escuelas.

En este sentido, podríamos considerar que, además de que la participación de los estudiantes campesinos en el movimiento universitario se advierte como un gesto de solidaridad en un momento en que su propia situación no era la más positiva, también es cierto que todos estos acontecimientos llegaron a caracterizar a esta década y la primera parte de los años setenta. Una época que para el caso de las normales rurales se inicia desde la primera mitad de los años sesenta cuando el propio José Santos Valdés identifica la persecución de que venían siendo objeto los estudiantes de estas escuelas y que culmina con el cierre de más de la mitad de escuelas de este tipo en el contexto de

41 Caja 34, Dirección General de Enseñanza Normal, Oficio 12656, A los padres de familia y estudiantes de la Sociedad de Alumnas de la Escuela Normal Rural de Cañada Honda, Ags., 18 de noviembre de 1968 .

42 También, Caja 34, Oficio 10434, Al Comité Ejecutivo de la Federación de Estudiantes Campesinos Socialistas de México, 4 de octubre de 1968.

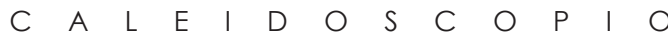


la reforma de educación normal de 1969. Una reforma que aunque parece no estar relacionada con el apoyo que otorgaron los alumnos de estas escuelas al movimiento universitario forma parte de un proyecto más complejo en el que, independientemente del protagonismo de estas escuelas, se aspiraba a atemperar a cualquier precio las inquietudes de las juventudes estudiantiles o de cualquier manifestación con tufo comunista.

Finalmente, al señalar que la participación de las normalistas rurales de Cañada Honda en torno al movimiento universitario se dio de diferentes maneras y reconociendo que no ocurrió de manera homogénea en todas las escuelas de este tipo en el país, nos encontramos ante un fenómeno que más que ayudarnos a concluir el tema nos coloca frente a nuevas interrogantes. Dado que las actividades desarrolladas en el contexto del movimiento universitario fueron sugeridas y coordinadas por la dirigencia nacional de la FECSM, como los paros de actividades o las movilizaciones y mítines desarrollados en el centro de la ciudad de Aguascalientes; nos llama la atención la descripción que hace una de las entrevistadas cuando se refiere a las guardias realizadas por las alumnas al interior de la escuela. Reconoce que el acuerdo de la base estudiantil era reunirse "todas las alumnas en las puertas para no dejar que entrara la policía o el ejército, en caso que se tratara de ellos" ${ }^{43}$ ¿Acaso una medida como ésta sería suficiente para evitar un desalojo? Si es que a lo largo de esos años las normalistas rurales venían luchando por sus propias demandas e incorporaron al movimiento universitario como un punto más de sus pliegos petitorios, ¿por qué el 2 de octubre dejó de ser un recuerdo y cómo se fue convirtiendo en un hecho presente para el normalismo rural mexicano?

\section{REFERENCIAS}

Aguayo Quezada, Sergio (2018). El 68. Los estudiantes, el presidente y la CIA. México: Ediciones Proceso.

Camacho Sandoval, Salvador (2003). Estudiantes y promoción cultural en el Aguascalientes sesentero. Aguascalientes: Universidad Autónoma de Aguascalientes, Documento sin publicar.

43 Palomino González, Mercedes (1964-1970). Entrevista realizada el 19 de mayo del 2018 en Villa Juárez, Ags., por Sergio Ortiz Briano. 
García Gómez, Daniel Carlos (2006). Fulgor Rebelde. La guerrilla en Aguascalientes. Ensayos. Aguascalientes: Filo de Agua. Colección Fuego Fresco. Instituto Cultural de Aguascalientes.

Guevara Niebla, Gilberto (2004). "Apéndice: Miembros del Consejo Nacional de Huelga". La libertad nunca se olvida. Memoria del 68, Ediciones cal y arena, pp. 327-333.

Rouquié, Alain (1987), citado en Victoriano Serrano, Felipe (2010).

"Estado, golpes de Estado y militarización en América Latina: una reflexión histórico política", en Argumentos (Méx.) Argumentos (Méx.) vol. 23 no. 64, México sep./dic.

Valdés García de León, José Santos (2005). Profr. José Santos Valdés, Centenario de su natalicio 1905-2005, Obras Completas. Tomo II, Educadores Democráticos de San Marcos, Zac., A. C.

SOBRE EL AUTOR

Sergio Ortiz Briano (sortiz_50@hotmail.com) es doctor en Historia por la Universidad Autónoma de Zacatecas. Se especializa en la historia de la educación en México, en particular en la historia de las escuelas normales rurales. Ha sido autor de libros como: Entre la nostalgia y la incertidumbre. Movimiento estudiantil en el normalismo rural mexicano (México: UAZ, 2012), así como numerosos artículos, entre los cuales se encuentra: "El normalismo rural mexicano y la 'conjura comunista' de los años sesenta. La experiencia estudiantil de Cañada Honda, Aguascalientes" (Revista Mexicana de Historia de la Educación: 5, no. 10, 2017). Actualmente se desempeña como profesor titular y responsable del área de Investigación Educativa en la Escuela Normal Rural "Justo Sierra Méndez" de Cañada Honda, Ags. (ORCID: 0000-00030632-3823). 